\title{
An Analysis of Forage Preference Indices
}

CRAIG LOEHLE AND LARRY R. RITTENHOUSE

Abstract

Of those models currently used to describe the preference of animals for various plants under given conditions, all have serious shortcomings for purposes of accurately explaining the data, in the regression sense. When five equations, based in various ways on preference and availability, were used to estimate diets of cattle and sheep, no clear advantage of one expression over another could be found. All models tested with the sheep data resulted in increased predicted sums of squares compared with total sums of squares. In contrast, models tested with the cattle data showed some reduction in unexplained variation in diet estimates during the entire year, spring, and summer, but not during fall. This improvement was probably because the cattle pastures were more homogeneous than the sheep pastures and species were aggregated. The best model was Ratio 4 (preference-availability) but it requires a complex and expensive parameter estimation technique. It was concluded that sampling problems combine with inadequacies of the preference indexes to prevent accurate representation of the concept of diet preference. It was also concluded that sampling problems arise when the fecal, rumen fistula, or esophageal fistual techniques are used to estimate diets. A technique for adjusting these techniques to make them suitable for predicting diets was described. Further investigations into animal behavior are needed to determine variables which affect what the animal perceives as being desirable in relation to what is available.

Preference indices in range work are used to summarize grazing trials, to draw conclusions about animal behavior (Krueger 1972), and to incorporate into diet prediction models (Nelson 1977, Nelson 1978) or simulation models (Anway 1976). However, the concept of preference has never been given a rigorous test. The objective of this paper is to approach such a test by analyzing the indices which purport to measure preference.

All models that could be located in the literature which incorporated preference were examined for usefulness in the context of large herbivores, particularly domestic stock. Those that passed logical examination were tested against grazing study data. Only one model (Silen and Dimock 1978) had been previously tested. None had been tested against data from natural pastures.

\section{Review of Previous Indices}

Many authors have developed indices of preference for animal diets. Some of the models developed for predation can be adapted to herbivory with suitable changes of definition.

Many of the models developed are limited to the 2-prey case. Chesson (1978) and Cock (1978) review and discuss many of these models. It is often stated (e.g., Cock 1978) that 2-prey models can be extended to the n-prey case by pooling all prey except type 1 into

Authors are research associate and associate professor, Department of Range Science, Colorado State University, Fort Collins. Colorado 80523.

Research was supported by the Colorado State Univ. Exp. Sta. and published as Sci. Series Pap. No. 2601 in cooperation with Regional Project W151.

The authors would like to express their appreciation to persons who reviewed this manuscript and for their many helpful suggestions. Further, we would like to thank Drs. Anderson and Krueger for having conducted such thorough studies and for allowing us to use their data for statistical analysis.

Manuscript received May 29, 1980.

the type 2 category and then treating as a 2-prey situation. This technique has never been validated and in fact is not tenable unless the pooled species a re equally preferred. Consider, for example, an animal (predator or herbivore) with 3 food items. Species 1 and 2 are greatly preferred but are much rarer than species 3 . Normally, the diet consists of equal parts of 1 and 2 plus a small a mount of 3 . If 2 and 3 are pooled into a new class $2^{i}$, the desirable characteristics of species 2 are overwhelmed by the undesirable character of 3 . This approach leads us to conclude, then, that the a nimal will eat almost entirely items of type 1 . The problem is that there is no tenable rationale for pooling species with different probabilities of encounter for herbivores or of capture for predators, frequencies in the habitat, and desirabilities to the predator (or herbivore). There are times when pooling species is unavoidable, but pooling into 2 classes as a standard procedure is an unjustified oversimplification. Similarly, pooling of plant parts may be an unjustified oversimplification (D. Swift, personal communication).

Because, in general, large herbivores consume more than two types of food, the many models that can represent only choices between two food items are not deemed useful. For these reasons such models will not be considered further in this paper.

Four relative preference indices (RPI's) are discussed by Krueger (1972). He rejects two of the indices because they do not meet the criterion that every plant has the same value when selection by animals is completely random. The models which pass Krueger's (1972) random grazer test are $R P_{1}$ and $R P_{2}$, which correspond to Ratio 1 and Ratio 2, discussed in the following section.

\section{Prediction Using Preference Ratios}

In this section, four preference ratio models are analyzed for logical validity. Those that meet this criteria are tested against data in the next section.

Ratio 1

Ratio $1(R 1)$ is the same as $R P_{1}$ (Krueger 1972) and is given by

$$
R l_{i}=\frac{\mathrm{fd}_{\mathrm{i}} \cdot \mathrm{D}_{\mathrm{i}}}{\mathrm{fr}_{\mathrm{i}} \cdot \mathrm{RA}_{\mathrm{i}}}
$$

where $R l_{i}$ is the value of Ratio 1 for the $i$ 'th plant species; $f d_{i}=$ frequency of species $i$ in the diet; $f_{i}=$ frequency of species $i$ in the pasture; $D_{i}=$ percent by weight of the diet for species $i$; and $R A_{i}=$ relative availability (percent by weight) of species $i$ in the pasture. Used as a predictive formula, we get

$$
\hat{D}_{i}=\frac{f r_{i}}{f d_{i}} \cdot R l_{i} \cdot R A_{i}
$$

This index has problems arising from the use of frequency. Because frequency depends on plot size and shape, and different management agencies and research workers use different sampling schemes, it would be very hard to get comparable results from place to place. In addition, the frequency of a food item to a herbivore depends on its mobility, the visibility of the food item under various conditions, and other factors. For these reasons, the usefulness of this index seemed dubious and it was not considered further. 


\section{Ratio 2}

This ratio has been used frcquently (Krueger 1972, Jacobs 1974, Cock 1978) and is commonly called the forage ratio in range work. It is given other names in other fields of ecology. It is described as the ratio of the percent of a species in the diet to the percent on the range. Its computational formula is

$$
\overline{\mathbf{P}}_{\mathrm{i}}=\sum_{\mathbf{k}=1}^{\mathrm{n}} \frac{\mathrm{D}_{\mathrm{ik}}}{\mathrm{RA}_{\mathrm{ik}}} / \mathrm{n}
$$

where $\bar{P}_{i}$ is the mean preference ratio over $n$ trials, $D_{i}$ is the percent of species $i$ in the diet, and $R_{i}$ is the percent of species $i$ in the herbage. The mean over $n$ trials is necessary due to the nature of the data (see methods). This model is easy to interpret in terms of whether an animal is selecting for or against a food item (species or species part).

The ratio can range from zero to infinity. In practice, infinite values are encountered when a rare species is found in the diet that was missed during vegetation sampling. This problem became apparent in this study during analysis of the Montana data. Once an infinite value is obtained, deriving a mean preference value is meaningless. In fact, large values, even if not infinite, can result from pasture heterogeneity and the difficulty of sampling rare species. In a statistical sense, conclusions drawn for such a rare species cannot be justified. The same conclusions apply to the log transform, below. This problem was addressed in this study by discarding all species that were rare enough to occur in the diet but not in the vegetation sampled.

Jacobs (1974) showed that by taking the log of the elements being summed in (3), symmetrical scales for positive and negative preference can be obtained. Kautz and Van Dyne (1978) used this formula:

let

$$
\mathrm{X}_{\mathrm{ik}}=\frac{\mathrm{D}_{\mathrm{ik}}+0.1}{\mathrm{RA}_{\mathrm{ik}}+0.1}
$$

then $\quad \ln \left(\bar{P}_{i}\right)=\frac{\sum_{k=1}^{n} \ln \left(X_{i k}\right)}{n}$

where the 0.1 term is added to avoid taking the log of zero or infinity. For more than one trial $(k>1)$, the symmetric nature of the scale seems to break down. It is not clear what meaning can be attributed to the results of all these manipulations. However, since the index $\tilde{P}_{i}$ in (5) was used in Kautz and Van Dyne (1978), it is tested here, along with the other indices.

Once the preference $\left(\overrightarrow{\mathrm{P}}_{\mathrm{i}}\right)$ is obtained from (3) or (5), the diet is estimated by

$$
\hat{\mathrm{D}}_{\mathrm{ik}}=\overrightarrow{\mathrm{P}}_{\mathrm{i}} \cdot \mathrm{RA}_{\mathrm{ik}} .
$$

In practice it was found that normalizing the diet estimates withın trials so that they summed to 1 improved the fit slightly. This practice was necessary because of the rare species which were excluded from consideration.

Manly et al. (1972) derived an instantaneous version of Ratio 2 that includes a preference factor and an estimation technique. Their method is limited to 2 species at present, which excludes it from further consideration.

\section{Ratio 3}

Ratio 3 (R3) is the same as the electivity index of Ivlev (1961). It is given by

$$
R 3_{i}=\sum_{k=1}^{n} \frac{D_{i k}-R A_{i k}}{D_{i k}+R A_{i k}} / n
$$

where symbols are as before. Compared to (3), (7) has the desirable property that it is symmetrical and bounded between -1 and 1 . Negative numbers indicate that the animal selects against a species; positive numbers indicate selection for the species.
Once $R 3_{i}$ has been obtained from (7) empirically, (7) can be treated as a single formula instead of a mean of ratios resulting in the predictive formula

$$
\hat{\mathrm{D}}_{\mathrm{ik}}=\frac{\mathrm{RA}_{\mathrm{ik}}\left(1+\mathrm{R} 3_{\mathrm{i}}\right)}{\left(1-\mathrm{R} 3_{\mathrm{i}}\right)} .
$$

A problem with this method is the fact that the diets predicted by (8) do not sum to 1 in all cases. Ratios derived for one pasture and applied to a similar one give diets that are reasonable but do not sum to 1 . Ratio 2 has the same problem, but we can justify normalizing the diets to sum to 1 in that case if we consider $\hat{D}$ in (6) as the joint probability of occurrence and selection. It is difficult to apply a similar argument to the normalization of $\hat{D}$ obtained from Ratio 3.

\section{Ratio 4}

A predictive model has been developed by Ellis et al. (1976) and used by Anway (1976). First, relative preference and relative availability indices were developed:

$$
R P_{i}=\frac{P_{i}}{\sum_{i} P_{i}} \quad R A_{i k}=\frac{A_{i k}}{\sum_{i} A_{i k}}
$$

where $P_{i}$ is preference for species $i$ and $A_{i}$ is availability of species $i$ in trial k. Preference was obtained from (3). The relative rank is then developed

$$
R R_{i k}=\frac{R P_{i} \cdot R A_{i k}}{\sum_{i}\left(R P_{i} \cdot R A_{i k}\right)}
$$

Ellis et al. (1976) then define the amount of food class $\left(C_{i}\right)$ consumed per unit time as

$\hat{C}_{i k}=(S)(C F R)\left(R_{i k}\right)+(1-S)(C F R)\left(R_{i k}\right)$

where $\mathrm{S}=$ proportion of food taken on a preference-density basis, $1-S=$ proportion of food taken strictly on an availability basis, CFR = total amount of food consumed in a unit time; if CFR = $100 \%$ or 1.0 , then $\hat{C}_{i}=\hat{D}_{i}$, as defined earlier. This model was tested with $R P_{i}$ in (10) derived by the method below. It was included because, while not strictly speaking a ratio index, it is still a simple formula closely related to preference.

If it is assumed that the animal takes no food based purely on availability, and that diet units are in percent, then the following equation results (Silen and Dimock 1978):

$$
\hat{D}_{i k}=\frac{R P_{i} \cdot R A_{i k}}{\sum_{i}\left(R P_{i} \cdot R A_{i k}\right)}
$$

Silen and Dimock (1978), however, do not use $R P_{i}$ derived from (9). Problems are encountered with the derivation of relative preference by methods such as Ratio 2 , as discussed in preceding sections. It is therefore desired to obtain $\mathbf{R} \mathbf{P}_{\mathrm{i}}$ directly from (11) or (12) in some manner.

Deriving preference values from (11) or (12) presents a number of statistical difficulties. The functions are non-linear so the usual regression techniques cannot be applied. In addition, the preference values do not truly represent parameters in the sense of the parameters of a function such as the negative binomial. The appropriate technique is therefore an iterative procedure that minimizes the difference between actual and predicted values for diet composition. This minimization procedure is subject to the constraint that the preference values must be greater than zero and sum to 1 . Such a technique was used in this study.

\section{Test of Preference Ratio Predictions}

\section{Methods}

A test was conducted with field data to see how well preference indices could explain data in the regression sense. Regression was not used to estimate preference indices because they are used in the 
literature as indices to be computed rather than as regression type models and because no single regression technique known to the authors would work for all models tested. An exception to this is Ratio 4. Thus variances of the parameter estimates could not be computed. Statistics were based on a goodness of fit approach. The null model was simply the mean percentage of each food item in the diet, the mean being computed over $n$ trials. Preference values were computed from the data, and then these values were used to back calculate predicted diets. Predicted diets were normalized to sum to one within each trial because of missing species. These predicted diets were compared to the actual percent of the food item in the diet, over all trials. If the sum of squares of predicted diets minus actual diets was less than the sum of squares of mean diets minus actual diets then the unexplained variation was considered to have been reduced, i.e., the preference index provided an informative summary of the data.

For both data sets, individual trials consisted of a fistula sample from one animal on a pasture for one day. In order to get a sufficient sample size, trials from several animals on several pastures had to be combined.

The test data consisted of sheep diets from a Montana mountain forb type obtained from Krueger (personal communication) and cattle diets from a midgrass type in Texas obtained from Anderson (1977).

Krueger's unpublished data were used in Krueger (1972). Diets were sampled from esophageally fistulated sheep for 1-hr collections shortly after sunrise. The vegetation was sampled with 30 plots $0.5 \mathrm{~m}^{2}$ on each pasture. Grazing trials consisted of 5 individual sheep grazing on several dates on each of several pastures. A total of 78 trials resulted. Over 37 species occurred in the diets, but $93 \%$ of the diets were accounted for by 14 species and $71 \%$ by 5 species. The 5 most common species comprised the test data set. Species not appearing in the vegetation but nevertheless appearing in the diet were excluded from consideration.

In Anderson's (1977) study, diets were sampled from esophageally fistulated cattle for 30 to 60 minutes shortly after sunrise. Animals grazed continuously and on a five-pasture rotation system (28 days graze and 120 days rest year-round). Availability was determined from $1001 / 4-\mathrm{m}^{2}$ plots in each pasture by a method similar to Edlefsen's (1960). Data were analyzed by season, i.e., spring, summer, or fall. Within each season, the original data had four categories; grasses, forbs, cactus (Opuntia spp.) and annual broomweed (Xanthocephalum spp.). The latter two were excluded from consideration in the analyses due to virtually complete unpalatability to cattle.

The forage ratio was tested by obtaining preference values from the data using (3) or (5) which was then put into (6) to obtain the diet estimate. The log transform (5) was only tested against the Texas data.

Electivity was tested with values from (7) being put into (8) to obtain the diet estimate.

The two models based on preference-availability (11) and (12)) were tested by obtaining preference values by non-linear optimization.

\section{Results}

Results of the tests of the predictive models are summarized in Tables 1 and 2 . Table 1 shows that all models tested against the of this model for all data sets and seasons tested suggest that it is the most promising of those tested.

squares for all seasons except fall (Table 2).

A closer examination of the sheep diet data (Table 1) revealed some trends. For all models the green weights gave slightly better predictions than the dry weights, although the significance of the difference was not tested. The reason for this is not clear. For both green and dry weights the best to worst models in a goodness of fit sense were preference-availability (12), electivity (8), and forage ratio (3), respectively. Because all of these models were unable to improve the prediction of the diets over the simple use of mean
Table 1. Analysis of selected models for sheep grazing in Montana, based on the five most common species, which comprise $71 \%$ of the diet (data from Krueger (1972)). Diet percentages obtained from (6) and (8) were normalized to sum to 1 for each trial within an experiment. Numbers are 100 times residual sum of squa res divided by total sum of squares for each experiment.

\begin{tabular}{llcc}
\hline \hline $\begin{array}{l}\text { Equation } \\
\text { number }\end{array}$ & Equation name & Dry weight & Green weight \\
\hline 3,6 & Forage ratio & 152.29 & 139.71 \\
8 & Electivity & 129.52 & 122.37 \\
12 & Preference- & 125.27 & 119.93 \\
& availability & & \\
\hline
\end{tabular}

proportions, it is probably not valid to consider the above ranking of model performances as meaningful.

In contrast to the Montana study, all models showed reasonable power to reduce variability in the Texas data (Table 2). Once again the preference-availability method was best, followed by the forage ratio. Electivity and the $\log$ transform of the forage ratio gave approximately equal results. The fall data did not always follow this trend and had a very poor fit, possibly because of the smaller number of trial dates within the fall season. For the entire year and for the spring, there was no difference in predictive power between the preference-availability and forage ratio methods. Nevertheless, the good fit with the preference-availability model obtained by Silen and Dimock (1978) and the consistently better performance of this model for all data sets and seasons tested suggest that is it the most promising of those tested.

Other results include further tests of the Montana data. The use of the 14 most common species, comprising $93 \%$ of the diet, resulted in much larger residual sum of squares than the 5-species test. No aggregation tests were tried. The model (11) of Ellis et al. (1976) was tested for 5 species, with $S$ (selectivity) varying from 0 to 0.9 by increments of 0.1 . Results of this model were always worse than the test of (12). Because of the poor fit of all models for the Montana data, it was not possible to say that (11) was invalidated by this result.

\section{Discussion}

There are two main causes of the lack of fit with the models tested: experimental design flaws relative to the diet prediction objective and lack of usefulness of the indices.

A major difference between the two data sets tested is pasture heterogeneity. The Texas pastures were relatively uniform and data were lumped into only 2 categories. In contrast, the Montana pastures were species rich and heterogeneous. No aggregation of the Montana data was attempted.

Spatial heterogeneity is important because a grazing animal makes choices largely on the basis of vegetation composition in its immediate area. Short grazing trials of a uniform pasture are clearly more likely to be representative of choices for an entire pasture than is a short grazing trial of a very heterogeneous pasture. The inference concerning spatial heterogeneity is confirmed when we consider the study of Silen and Dimock (1978),

Table 2. Analysis of selected models for cattle grazing in Texas (data from Anderson (1977)). Diet percentages obtained from (6) and (8) were normalized to sum to 1 for each trial within a season. Numbers are 100 times residual sum of squares divided by total sum of squares for trials over the respective seasons.

\begin{tabular}{|c|c|c|c|c|c|}
\hline $\begin{array}{l}\text { Equation } \\
\text { number }\end{array}$ & Equation name & $\begin{array}{l}\text { Entire } \\
\text { year }\end{array}$ & Spring & Summer & Fall \\
\hline$\overline{3,6}$ & Forage ratio & 55.20 & 71.89 & 56.07 & 278.22 \\
\hline 5,6 & $\begin{array}{l}\text { Forage ratio ( } \mathrm{Log} \\
\text { transform) }\end{array}$ & 63.03 & 72.97 & 66.05 & 193.34 \\
\hline 8 & Electivity & 62.28 & 73.38 & 64.44 & 195.98 \\
\hline 12 & Preference-availability & 55.10 & 71.61 & 46.77 & 189.41 \\
\hline
\end{tabular}


who used (12) to model damage patterns to tree seedlings by deer and hares. Their results were statistically highly significant. They counted damage to seedlings on a 24-hour basis. Because no trial had more than 144 seedlings, this gave the hare or deer sufficient time to make choices from the entire population of plants, particularly as the number undamaged decreased. In contrast, a large herbivore grazing for one hour while filling an esophageal fistula bag, cannot adequately cover most pastures. Natural pastures are quite heterogeneous, and the vegetation covered by a herbivore during one hour may not be very representative of the "average" vegetative composition as sampled by plots or transects. A larger number of fistula samples averaged together, or a longer sample period, such as obtained with rumen or fecal sample techniques, would be more likely to result in diet estimates that can be compared with the total pasture species composition [the assumption being that biases inherent in these techniques can be accounted for].

The length of the sample period or number of samples sufficient to obtain a representative diet needs to be determined experimentally. Increasingly long sample periods could be tested until relatively constant percentages of species in the diet are obtained. For example, for day 1 the fecal (or esophageal or fistular) sample variation between animals is obtained. On day 2 feces are obtained and pooled in equal proportions with that left over from day 1 from the same animal to obtain a 2-day-long sample. This procedure is repeated until the between-animal variation reaches a relatively constant minimum. This same technique could be used for rumen or esophageal fistula samples, for example, by comparing 10,15 , etc., minute fistula samples until a constant diet per animal or between animals was obtained up to the physical collection time limit, often less than 1 hour.

Another aspect of animal behavior that affects measures of availability is the relationship of grazing time with distance from fences or water and steepness of slope. On large and/or hilly pastures, in particular., it is hard to justify the exclusion of such factors from consideration unless the pasture is very homogeneous. It is possible to include factors affecting animal distribution by constructing a probability density function for occupation of parts of the pasture based solely on physical factors such as slope steepness and distance from water or fences (Senft, et al. 1980). The correlation of plant species with topography is a possible confounding factor. To correct for this, when the vegetation is sampled, each species on each sample plot can be weighted by the probability of the a nimal being found in that part of the pasture. These weighted availabilities are likely to be quite different from the mere sum of biomasses over the pasture. This procedure might require more plots to obtain a stable estimate of species proportions and abundances but would likely eliminate a great deal of the bias inherent in the current random sampling methods.

Assessing the role of the models in the failure to fit the data is difficult because of the experimental design problems discussed above. The magnitude of the experimental design problem becomes apparent when a comparison is made between the highly significant results for (12) obtained by Silen and Dimock (1978) and the residual sum of squares values obtained with (12) for the Texas data. Whether diets on many-species pastures can be adequately explained with the models tested cannot be stated until the sampling problems caused by spatial heterogeneity are resolved.

The problems inherent in the esophageal fistula technique discussed above led to the examination of other aspects of sampling vegetation for diet analysis or prediction. Availability to the animal is usually measured strictly in terms of standing crop per hectare based on $1 \mathrm{~m}^{2}$ (or other size) plots. There are a number of reasons to suspect the general usefulness of this approach. Consider that domestic stock do not know the biomass of all species on a per hectare basis except relatively and approximately on small pastures. We should be measuring variables relevant to the grazer's perception of availability if we hope to make any useful predictions. For example, plants or plant parts that grow closer to the ground than the animal can graze are not available to the grazer and should not be counted in the total forage per hectare. Another problem was pointed out by Arnold (1963), who showed that preferred green forage may be completely inaccessible at certain seasons because of dense standing dead material. Simply clipping the available forage on sa mple plots is obviously misleading in such cases. The same problems arise when measuring frequency. A plant should not be counted as occurring on a plot if it is not available to the animal.

It is not clear in any case that standing crop is the variable of interest to the grazer. Tall plants are more visible and may be perceived as more abundant by the grazer. Other factors, such as cover, leaf size, frequency, moist ure content, sugars, protein, etc., may also be important. The validity of using standing crop as the sole independent variable needs to be demonstrated and cannot be assumed a priori.

The role of the indices themselves in the failure to explain the data is difficult to ascertain. The concept of preference makes intuitive sense and is therefore difficult to abandon. The results of this study, however, show the danger of using indices or models without ever testing their validity.

\section{Bibliography}

Anderson, D.M. 1977. Standing crop, diets, travel and weight changes under short-duration and continuous grazing. Ph.D. Thesis, Texas A\&M Univ., College Station. 181 p.

Anway, J.C. 1976. Mammalian consumer submodel, p. 137-195. In: G.W. Cole (ed.) ELM: Version 2.0. Range Sci. Dep. Sci. Ser. No. 20. Colorado State Univ., Fort Collins.

Arnold, G.W. 1963. Factors within plant associations affecting the behavior and performance of grazing animals, p. 133-154. In: D.J. Crisp (ed.) Grazing in terrestrial and marine environments.

Chesson, J. 1978. Measuring preference in selective predation. Ecology 59:211-215

Cock, M.J.W. 1978. The assessment of preference. J. Anim. Ecol. 47:805816.

Edlefsen, J.L., C.W. Cook, and J.T. Blake. 1960. Nutrient content of the diet as determined by hand plucked and esophageal fistula samples. J. Anim. Sci. 19:560-567.

Ellis, J.E., J.A. Wiens, C.F. Rodell, and J.C. Anway. 1976. A conceptual model of dict selection as an ecosystem process. J. Theor. Biol. 60:93-108.

Ivlev, V.S. 1961. Experimental ecology of the feeding of fishes. Yale Univ. Press.

Jacobs, J. 1974. Quantitative measurement of food selection. A modification of the forage ratio and Ivlev's electivity index. Oecologia (Berlin), 14:413-417.

Kautz, J.E., and G.M. Van Dyne. 1978. Comparative analyses of diets of bison, cattle, sheep, and pronghorn antelope on shortgrass prairie in northestern Colorado, U.S.A., p. 438-443. In: D.N. Hyder (ed.) Proc. 1st Int. Rangeland Congr., Soc. Range Manage.

Krueger, W.C. 1972. Evaluating animal forage preference. J. Range Manage. 25:471-475.

Manly, B.F., J.P. Miller, and L.M. Cook. 1972. Analysis of a selective predation experiment. Amer. Natur. 106:719-736.

Nelson, Jack R. 1978. Maximizing mixed animal species stocking rates under proper-use management. J. Wildl. Manage. 42:172-174.

Nelson, Jack R. 1977. Maximizing stocking rates with common-use and proper-use grazing. Washington State Univ., College of Agr. Res. Center Bull. 856

Senft, R., L.R. Rittenhouse, and R. Woodmansee. 1980. Predicting patterns of cattle behavior on shortgrass prairie. Proc. West. Sec. Amer. Soc. Anim. Sci. 31:276-279.

Silen, R.R., and E.J. Dimock II. 1978. Modeling feeding preferences by hare and deer among Douglas-fir genotypes. Forest Sci. 24:57-64. 